\title{
PRACTICE
}

- Cardiovascular disease is common

- Pain and anxiety increase cardiac load and increase the risk of precipitating angina/ arrhythmias

- A thorough history will usually elicit the fact that the patient has cardiovascular disease (summarised in Table 2)

- Examination of the patient may reveal cardiovascular disease - cyanosis (central/peripheral), shortage of breath, abnormalities in the pulse, finger clubbing, splinter haemorrhages or ankle oedema

- Drugs used in the treatment of cardiovascular disease impact on patient management

\section{General medicine and surgery for dental practitioners Part 1: Cardiovascular system}

\author{
M. Greenwood ${ }^{1}$ and J.G. Meechan ${ }^{2}$
}

This series examines aspects of general medicine and surgery which are of relevance to dental practice. The approach is standardised by considering systems under common headings eg history, examination, commonly prescribed drugs and aspects relating to general and local anaesthesia and management in the dental surgery. First in the series is a consideration of the cardiovascular system.

GENERAL MEDICINE AND SURGERY FOR DENTAL PRACTITIONERS:

1. Cardiovascular system

2. Respiratory system

3. Gastrointestinal system

4. Neurological disorders

5. Liver disease

6. The endocrine system

7. Renal disorders

8. Musculoskeletal system

9. Haematology and patients with bleeding problems

10. The paediatric patient
${ }^{1}$ Lecturer, ${ }^{2}$ Senior Lecturer, Department of Oral and Maxillofacial Surgery, The Dental School, Framlington Place, Newcastle upon Tyne NE2 4BW Correspondence to: M. Greenwood E-mail:beryl.leggatt@ncl.ac.uk

\section{Refereed Paper}

๑ British Dental Journal 2003; 194

537-542
Cardiovascular disease is common and it is inevitable that any practitioner dealing with patients will encounter it. In 1984 it was estimated that $2 \%$ of all adult dental patients were receiving anti-hypertensive therapy. ${ }^{1,2}$ This figure has risen and in 1997 it was reported that up to $13 \%$ of patients in a dental hospital setting and 5\% of those attending dental practice were receiving anti-hypertensive drugs. ${ }^{3}$ There may be a well-established previous history of cardiovascular disease. The incidence increases with age such that, by the age of 70, all patients will have some degree of cardiovascular disease (this may be very minor and subclinical or the origin not recognised by the patient eg calf claudication, a sign of peripheral vascular disease).

Risk factors for cardiovascular disease are shown in Table 1.

In the history it is clearly important to assess the degree of compensation that the patient has managed to achieve, ie how badly the patient is

\begin{tabular}{l} 
Table 1 Risk factors for \\
cardiovascular disease \\
\hline - Smoking \\
- Excess alcohol \\
- Diabetes mellitus \\
- Hypercholesterolaemia \\
- Family history of cardiovascular \\
disease \\
- Sedentary lifestyle \\
- Obesity
\end{tabular}

affected by their condition in terms of signs, symptoms and activity. The efficacy of medication is also important. Some patients may be taking aspirin on a regular basis. Specific enquiry is important because of aspirin's effects on blood clotting.

\section{RELEVANT POINTS IN THE HISTORY}

Other points to ask in the history (Table 2) include the following:

\section{Chest pain}

The purpose of questioning here is not to try to be diagnostic but to gain an idea as to whether a cardiovascular cause for the pain may be likely, since some patients may be unaware of their condition but nevertheless be at risk.

Features which make the pain unlikely to be cardiac in origin are: pains lasting less that 30 seconds however severe, stabbing pains, welllocalised left submammary (under the breast) pain, and pains which continually vary in location. A chest pain made better by stopping exercise is more likely to be cardiac in origin than one that is not related (see Myocardial Infarction, Angina). Pleuritic pain is sharp and made worse on inspiration, eg in pulmonary embolism. Shingles (varicella zoster) may cause pain following a particular nerve territory. The characteristic rash is preceded by an area of hyperaesthesia.

Oesophagitis may cause a retrosternal pain which is worse on bending or lying down. However, oesophageal pain, like cardiac pain, may be 


\begin{tabular}{l} 
Table 2 Relevant points in the \\
history with reference to \\
the cardiovascular system \\
\hline - Chest pain \\
- Angina \\
- Myocardial infarction \\
- Hypertension \\
- Medication eg aspirin, warfarin \\
- Syncope \\
- Shortage of breath/ \\
- exercise tolerance \\
- Rheumatic fever \\
- Cardiac rate/rhythm \\
- Cardiomyopathy \\
- Coronary artery bypass graft \\
- Valve replacements \\
- Congenital disorders \\
- Cardiac transplants \\
- Venous/lymphatic \\
disorders
\end{tabular}

relieved by sublingual nitrates, eg glyceryl trinitrate (GTN).

Hyperventilation may produce chest pain. Gallbladder and pancreatic disease may also mimic cardiac pain. Musculoskeletal pain is often accompanied by tenderness to palpation in the affected region.

\section{Angina pectoris}

This central, crushing chest pain may radiate to the neck, mandible and one or both arms. It may be felt in only one of these sites. Unstable angina is that occurring at rest, minimal exertion or with rapidly increasing severity. There is a significant risk of myocardial infarction and elective surgery should not be carried out on the patient with unstable angina. When performing emergency treatment on such patients the use of epinephrine (adrenaline) containing local anaesthetics is best avoided. ${ }^{4}$ The severity of angina may be gauged by the exertion required to provoke an attack, and the efficacy of medication to induce relief.

Effective analgesia, short appointments, availability of oxygen and GTN are all important in treatment regimens. The use of sedation should be considered in these patients as an added stress reduction measure. GTN should relieve chest pain in angina within 5 minutes. A spray formulation is now commonly used; this is the preferred formulation as the emergency medicament in practice as it has a longer shelflife than the tablet formulation (once the bottle has been opened).

\section{Myocardial infarction (MI)}

The signs and symptoms of MI are well known and may be like angina but more severe and of longer duration. Importantly, it is not relieved by GTN. Some myocardial infarctions are 'silent', ie occur with no recognised symptoms or signs at the time. The residual deficit is a marker of severity of the original event. Admission to hospital, the coronary care unit and duration of admission are also indicative.

The timings for dental treatment, for both local and general anaesthesia post MI, are given later, but in all cases obviously local analgesia must be maximally effective, and GA carried out in a hospital environment. As mentioned above sedation should be considered for many of these patients.

\section{Hypertension}

There is variation but, in general terms, corrective treatment is carried out if the blood pressure is persistently more than 200 Systolic or over 110 Diastolic. Treatment may be indicated at lower levels if vascular complications are evident.

Most hypertension is 'essential' (90\%), ie no cause found. The aim of treatment is to maintain a blood pressure less than 160/90. Stress may further increase an already raised blood pressure, leading to risk of stroke or cardiac arrest.

Postural hypotension, eg on suddenly rising from the supine position or rapid alteration of the dental chair, may be a side effect of some antihypertensive drugs. There may be underlying cardiac or renal disease in some patients with hypertension. Many antihypertensive drugs impact on dental management (see later).

\section{Syncope or fainting}

This, as is well known, may be precipitated by fear and may be vasovagal or cardiac in origin. Respiratory syncope (in cases of extreme coughing bouts) also exists.

In the 'carotid sinus syndrome', mild pressure on the neck causes syncope with bradycardia or cardiac arrest.

\section{Shortage of breath (SOB)/exercise tolerance}

SOB is often a sign of cardiac failure, but must be differentiated from respiratory disease with which, of course, it may co-exist.

The degree of severity can be assessed by enquiring about whether the patient ever wakes up in the night with breathlessness (paroxysmal nocturnal dyspnoea), or has orthopnoea, ie becoming breathless on lying flat at night. The degree of exertion needed to precipitate breathlessness is also important.

In uncontrolled cardiac failure, dental treatment under any form of anaesthesia should be deferred until medication and symptoms are stabilised. Even when relatively well-controlled, putting the patient in the supine position may exacerbate dyspnoea and is therefore best avoided. Cor Pulmonale is the term used to describe heart failure secondary to pulmonary disease, caused by an excess load on the right ventricle.

In 'left-sided heart failure', the oedema is pulmonary, whereas in 'right-sided heart failure' it is peripheral (sacral in the bed-bound and ankles in the ambulant).

\section{Rheumatic fever}

There may not be any subsequent cardiac damage, but this can only be determined definitively by a cardiologist. These patients may be more at risk of life-threatening reactions to prophylactic antibiotics compared with the development of infective endocarditis (see later). ${ }^{5} \mathrm{~A}$ typical rash in a patient allergic to penicillin who has taken the antibiotic is shown in Figure 1.

The degree of risk of infective endocarditis is

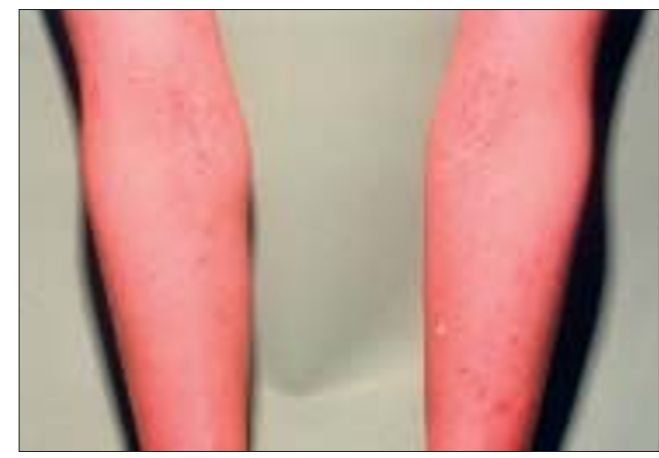

Fig. 1 A typical rash on the forearms of a patient who has recently been given oral penicillin for prophylaxis against infective endocarditis 
not directly related to the degree of damage to a heart valve. Dental treatment during the acute phase of rheumatic fever should be performed only after consultation with a physician.

\section{Infective endocarditis}

This is uncommon and may be acute or chronic. The disease may affect damaged heart valves, prosthetic heart valves, a coarctated aorta, patent ductus arteriosus or ventricular septal defect.

The viridans Streptococci are the most commonly isolated bacteria. In the United Kingdom dental patients are defined as at 'special risk' of developing infective endocarditis if they have a previous history of infective endocarditis, or a prosthetic heart valve and are having treatment under general anaesthesia. Risk assessment varies between countries. ${ }^{5}$

It is important to ascertain whether there is a previous history of heart murmur, ${ }^{6}$ history of rheumatic fever or valve problems. Surgically constructed shunts are a risk. It is also important to assess whether there has been previous infective endocarditis.

Syndromic patients, eg those with Down Syndrome, should be suspected of cardiac involvement. Individual congenital abnormalities often appear in association. Other causes of infective endocarditis include drug abuse such as heroin addiction. ${ }^{7}$

Antibiotic 'cover' is not required post myocardial infarction, coronary artery bypass graft (CABG) or after 6 months post atrial septal defect repair without a Dacron patch. Atrial septal repair with a Dacron patch constitutes a risk factor for infective endocarditis and therefore prophylactic antibiotics are required for procedures likely to cause a bacteraemia. Patients who are 6 months after a repaired patent ductus arteriosus or who have had a cardiac transplant more than 6 months ago do not require prophylactic antibiotics. A permanent cardiac pacemaker likewise does not require antibiotic prophylaxis for invasive procedures likely to cause a bacteraemia. Patients with diagnosed pulmonary stenosis do not require antibiotic prophylaxis.

Regimens for antibiotic prophylaxis are to be found in the current British National Formulary and procedures requiring antibiotic prophylaxis are listed in Table 3.

\section{Cardiac rate/rhythm}

The patient may give a history of palpitations or an established history of arrhythmia. They may have a pacemaker.

Pacemakers may be temporary or permanent. Care needs to be taken with electrical equipment which can unbalance the circuits within a pacemaker. Magnetic resonance imaging (MRI) scanners, electrosurgery and diathermy can all be problematical, as can ultrasonic scalers. Electric pulp testers do not present a risk.

Temporary pacemakers may necessitate antibiotic prophylaxis for the procedures in Table 3 and the physician responsible should be consulted.
Table 3 Procedures requiring antibiotic prophylaxis

- Dental extractions

- Any procedure involving the raising of a mucosal/ mucoperiosteal flap

- Biopsies

- Any subgingival procedure eg placement of orthodontic bands (not brackets), scaling of teeth, irrigation of periodontal pockets

- Intraligamentary injections

- Reimplantation of avulsed teeth

- Incision and drainage of an abscess

- Placement of dental implants

- During diagnostic phase of root canal therapy if it is thought likely that an instrument may pass through the tooth apex

Table 4 Common arrhythmias which may be encountered in dental practice

- Sinus Tachycardia - pulse over 100 beats per minute

- Sinus Bradycardia - pulse less than 60 beats per minute

- Atrial Fibrillation - totally irregular wrist pulse

- Ventricular Extrasystole - 'missed beats' at the wrist

\section{COMMON ARRHYTHMIAS (see Table 4)}

Sinus tachycardia (the pulse is more than 100 beats per minute)

This may be physiological (exercise, emotion, anxiety, pain) or be related to fever, post myocardial infarction, shock, heart failure and with some drugs (epinephrine, atropine). Hyperthyroidism, smoking and excessive coffee ingestion may also be causes.

\section{Sinus bradycardia (the pulse is less than 60 beats per minute)}

This may occur physiologically in athletes or in vasovagal attack. Drugs such as beta blockers or digoxin may cause it. Post myocardial infarction and the 'sick sinus syndrome' may all be causative, as may hypothyroidism.

\section{Atrial fibrillation}

This is common in the elderly and may be asymptomatic. An irregularly irregular pulse is palpable at the wrist. If a wrist pulse is palpated eg after a faint or during sedation, it will frequently be encountered as a pre-existing anomaly.

\section{Ventricular extrasystole}

This is the commonest arrhythmia after a myocardial infarction. Three successive extrasystoles are described as ventricular tachycardia. An extrasystole is an 'extra' ventricular contraction. A ventricular extrasystole may be felt as a 'missed beat' at the wrist. They are usually of no significance.

Arrhythmias are relevant since they may be exacerbated by dental treatment caused by the associated stress, or by general anaesthesia. ${ }^{8}$ Common arrhythmias are summarised in Table 4. Arrhythmias may be increased by manipulation of eyes, carotid sinus or neck by pathways mediated by the vagus nerve.

\begin{tabular}{l} 
Antibiotic \\
prophylaxis \\
\hline Antibiotic \\
prophylaxis is \\
required to \\
prevent infective \\
endocarditis for \\
patients with certain \\
cardiac conditions. \\
Not all cardiac \\
conditions need \\
antibiotic prophylaxis
\end{tabular}




\section{PRACTICE}

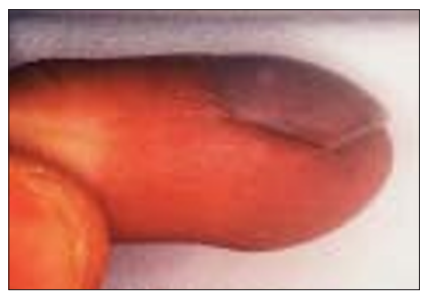

Fig. 2 Finger clubbing. This patient also demonstrates peripheral cyanosis
Table 5 Congenital cardiac defects may be broadly divided into cyanotic and acyanotic categories

\begin{tabular}{ll}
\hline Cyanotic & Acyanotic \\
\hline Transposition of & Ventricular septal defect \\
great vessels & Atrial septal defect \\
Fallot's tetralogy - & Patent ductus arteriosus \\
(Ventricular septal & Aortic coarctation \\
defect & \\
Pulmonary stenosis & \\
Right ventricular hypertrophy & \\
Overriding aorta) &
\end{tabular}

\section{Cardiomyopathy}

This is a general term meaning disease of the heart muscle. These patients (who may well be unaware of the condition) may be at increased risk from infective endocarditis in the idiopathic or hypertrophic cases and consultation with their cardiologist is important.

\section{Coronary artery bypass graft}

Exercise tolerance and history of chest pain should be enquired about post-bypass.

\section{Valve replacement}

Artificial valves may be tissue or mechanical. The latter patients are placed on life-long warfarin. Patients with prosthetic heart valves require antibiotic cover for dental procedures which produce bacteraemia.

\section{Congenital cardiac defects}

Congenital cardiac defects may be divided into cyanotic or acyanotic types. In the former, chronic hypoxaemia leads to finger clubbing (Fig. 2) and polycythaemia. The polycythaemia may lead to a tendency to haemolysis or thrombosis. The disorders fitting into the broad categories are shown in Table 5.

Infective endocarditis risk and bleeding tendencies are the most relevant factors from the dental standpoint. Cerebral abscess is a risk in these patients.

\section{Cardiac transplants}

Pre-operatively, it is important to eradicate potential or actual sources of infection and to optimise oral hygiene. Such patients will usually be treated in the hospital setting.

\begin{tabular}{|c|c|}
\hline \multirow[t]{4}{*}{ - Systemic Causes: } & Congestive cardiac failure \\
\hline & 'Right heart' failure \\
\hline & Hypoalbuminaemia eg nephrotic syndrome \\
\hline & Fluid overload \\
\hline \multirow[t]{2}{*}{ - Regional Causes: } & Venous obstruction eg advanced pregnancy \\
\hline & Lymphatic obstruction \\
\hline \multirow[t]{5}{*}{ - Local: } & Sluggish venous return eg poor muscle pump \\
\hline & in a paraplegic patient \\
\hline & Acute obstruction to venous return \\
\hline & eg DVT, Previous DVT \\
\hline & Cellulitis, Iymphatic aplasia/obstruction \\
\hline
\end{tabular}

Post-transplant treatment may be complicated by:

- Immunosuppression

- Steroid therapy

- Risk of infective endocarditis (in the first 6 months)

- Gingival overgrowth as a result of post-transplant drug therapy ${ }^{9}$ (Fig. 3)

- Supersensitivity of the transplanted heart to circulating catecholamines ${ }^{10}$ which may include epinephrine in dental local anaesthetics ${ }^{11}$

- Hepatitis, HIV Infection (rarely)

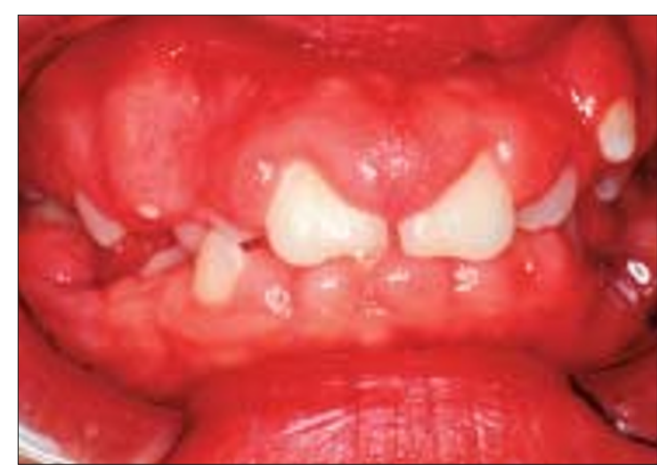

Fig. 3 Gingival hyperplasia in a post-cardiac transplant patient who is taking the calcium antagonist nifedipine

\section{Venous/lymphatic disorders}

A swollen limb may be a sign of heart failure. Causes of a swollen limb may be divided into systemic, regional and local as shown in Table 6.

For dental purposes, the patient should be treated with legs elevated to minimise dependent oedema, but the practitioner should beware of orthopnoea.

\section{EXAMINATION}

The most obvious initial observations are those of the patient's general demeanour, colour, whether short of breath at rest (SOBAR), or on minimal exertion, eg walking into the surgery (obviously primary respiratory causes may also exist). SOBAR indicates severe cardiorespiratory disease. A pulse oximeter reading is a useful rough guide to the efficiency of ventilation.

Cyanosis may be central, eg lip, tongue, or peripheral, eg nail beds (Fig. 2). Cyanosis represents a concentration of desaturated haemoglobin of at least 5 grams per decilitre. The pulse in terms of rate, volume, rhythm and character can give important clues to the state of the cardiovascular system and, indeed, other systems.

Clubbing of the fingers (loss of the angle between nail and nail bed when a finger is viewed from the side) may occur in infective endocarditis, cyanotic congenital heart disease and thyrotoxicosis (in which atrial fibrillation may also be noted).

Splinter haemorrhages are vasculitic consequences of infective endocarditis visible in the nail beds. Osler's Nodes may also occur (painful lesions on the finger pulps) and mac- 
ules on the palms (Janeway Lesions) in infective endocarditis.

Swollen ankles may be a sign of cardiac failure and oedema occurs in the sacrum in bedbound patients.

\section{DRUGS USED IN CARDIOVASCULAR DISEASE}

\section{Beta blockers}

These drugs decrease the sympathetic effects on the cardiovascular system, eg atenolol, propranolol, sotalol. Beta-blockade inhibits any reduction in diastolic blood pressure produced by epinephrine in dental local anaesthetics ${ }^{12}$ which might result in an uncompensated rise in systolic blood pressure. Thus dose limitation of epinephrine is wise when patients are taking beta blockers, two cartridges of an epinephrine containing solution in an adult is a sensible limit.

Oral side-effects can include dry mouth and lichenoid reactions.

\section{Diuretics}

These may be used in hypertension (thiazides only) and heart failure. Patients receiving non potassium sparing diuretics have been shown to experience an increased hypokalaemic response to epinephrine in dental local anaesthetics compared to healthy patients ${ }^{13}$ and this could predispose to arrhythmias. A limit of one to two epinephrine containing LA cartridges is recommended.

\section{Digoxin}

This is used to slow the ventricular rate in fast atrial fibrillation. The old-fashioned use is in the treatment of heart failure - Angiotensin Converting Enzyme (ACE) Inhibitors are now more commonly used (see later).

\section{Vasodilators}

ACE Inhibitors Renin, produced by the kidney, converts Angiotensinogen to Angiotensin I, which is converted in the lungs by Angiotensin Converting Enzyme (ACE) to Angiotensin II. Angiotensin II stimulates the adrenal cortex to produce Aldosterone which induces peripheral vasoconstriction. Aldosterone activates the pump in the distal renal tubule leading to reabsorption of sodium and water from urine, in exchange for potassium and hydrogen ions.

ACE Inhibitors may induce angioedema and lichenoid reactions, there may be taste loss with enalapril and captopril. Erythema Multiforme may also be induced. Burning mouth has also been reported. NSAIDs should be avoided as the risk of renal damage is increased.

Other vasodilators decrease the blood pressure in hypertension. This decreases the work of the heart in cardiac failure. They may dilate predominantly veins, eg nitrates, or arteries eg hydralazine, or a mixture eg prazosin.
Calcium antagonists. These cause coronary and peripheral vessel vasodilation and are negatively inotropic ie they reduce the strength of cardiac contraction. They are antiarrhythmic. Calcium antagonists are used in coronary heart disease and hypertension. Examples include nifedipine and diltiazem.

Oral side-effects include gingival hyperplasia. ${ }^{9}$ Headache and flushing may occur, as can peripheral oedema.

\section{Warfarin}

This may be used in the management of atrial fibrillation (as thromboembolic prophylaxis), deep vein thrombosis (DVT), prevention of embolisation secondary to MI and after prosthetic heart valve replacement.

The therapeutic efficacy is monitored using the International Normalised Ratio (INR). There are local variations in what is considered to be a 'safe' INR to carry out surgical dental treatment. This aspect is discussed fully later under bleeding disorders in this series.

The INR should be checked on the day of the procedure. Whenever warfarin dosage is adjusted, the normal regimen is to stop the drug two days before the procedure, with an INR check pre-operatively and resumption of the warfarin on the evening of the day of procedure. Adjustment must be in consultation with the patient's physician.

\section{Heparin}

This is an anticoagulant usually used in the hospital setting. It is monitored by the Activated Partial Thromboplastin Time (APTT).

Since the advent of the low molecular weight heparins, some cases of DVT are now treated on a community basis and a dental surgeon in practice could encounter a patient on this form of treatment. Tinzaparin and enoxaparin are two of the more commonly used agents; they have little effect on dental treatment.

\section{GENERAL AND LOCAL ANAESTHESIA, SEDATION AND MANAGEMENT CONSIDERATIONS IN THE DENTAL PATIENT WITH CARDIOVASCULAR DISEASE}

As alluded to earlier, the key to assessment is the degree of compensation or control of the underlying disorder that has been achieved. The relevance with regard to anaesthesia of some disorders is discussed earlier. The American Society of Anaesthesiologists (ASA) has developed a system known as the ASA Classification, which is a universally recognised stratification of patient

\begin{tabular}{ll} 
Table 7 American Society of Anaesthesiologists (ASA) Classification \\
\hline ASA I & Healthy \\
ASA II & Mild systemic disease - No functional limitation \\
ASA III & Severe systemic disease - Definite functional limitation \\
ASA IV & Severe disease - Constant threat to life \\
ASAV & Moribund
\end{tabular}

Drugs

Drugs used in cardiovascular disease affect the choice of local analgesia, other analgesics and may affect bleeding 


\begin{tabular}{lc} 
Table 8 Prognosis after MI with general anaesthesia \\
\hline Time since infarction & $\begin{array}{c}\text { Incidence of further } \\
\text { infarction after surgery (\%) }\end{array}$ \\
\hline $0-6$ months & 55 \\
1-2 years & 22 \\
2-3 years & 6 \\
$>3$ years & 1 \\
No Infarction & 0.66
\end{tabular}

fitness (encompassing all systems of the body). The classification is shown in Table 7.

In hypertensive patients, if feasible, treatment is best carried out under local analgesia, with or without sedation. As mentioned previously both beta-blocking and non-potassium sparing diuretic drugs can exacerbate unwanted effects of epinephrine in dental local anaesthetics and dose reduction of epinephrine is wise. Similarly, patients who have had cardiac transplants may super-react to the cardiac effects of epinephrine in dental local anaesthetics. The use of sedation may be valuable in patients with cardiac disease. Firstly, sedation may reduce the effects of stress. Secondly, the use of sedation may eliminate the need for general anaesthesia. Antihypertensive drugs are not usually stopped before a general anaesthetic.

In patients post myocardial infarction, elective surgery under GA or LA should be postponed for at least 3 months and, ideally, a year. Within 3 months of an MI, even emergency treatment is best carried out with medical consultation. The prognosis after an MI of patients undergoing a general anaesthetic is shown in Table 8.

Aspects relating to the management of patients with cardiovascular disease other than operative pain control measures, include the treatment of conditions secondary to drug therapy and post-operative pain control. Drug problems which may arise include dry mouth which will necessitate a preventive regimen and when severe may require the use of artificial saliva. Drug-induced gingival overgrowth can occur as mentioned earlier as a result of post-transplantation drugs and calcium-channel blockers (Fig. 3). Repeated gingival surgery is not uncommon in such patients.

Normally, post-operative pain in dentistry is controlled by non-steroidal analgesics. However, the use of non-steroidal anti-inflammatory drugs such as aspirin should be avoided in patients taking warfarin as the anti-coagulant effect is increased. Similarly, non-steroidal drugs inhibit the hypotensive effects of anti-hypertensive medication and their nephrotoxicity is increased in the presence of diuretics.
Smoking is a common cause of peri-operative morbidity in the context of GA. In addition to its deleterious respiratory effects, the carbon monoxide produced by cigarettes has a negatively inotropic effect. Nicotine increases the heart rate and systemic arterial blood pressure.

Carbon monoxide decreases oxygen supply and nicotine increases oxygen demand. This is particularly significant in patients with ischaemic heart disease. These patients can get real benefit by stopping smoking 12-24 hours before surgery. The negative respiratory effects of smoking take at least 6 weeks to start to abate.

\section{SUMMARY}

There are many factors which need to be borne in mind from the cardiovascular point of view when assessing the status of a patient requiring dental treatment. The degree of control of the disease, sequelae arising from it and time from the causative event can all be of importance in treatment planning. Much of the information required to make safe decisions will be obtained through a thorough history.

The authors would like to thank Professor J. V. Soames and Prof R. R. Welbury for providing clinical photographs.

1. Hemsley S M. Drug therapy in dental practice. Br Dent J 1984; 157:368.

2. Punnia Moorthy A, Coghlan K, O'Neil R. Drug therapy among dental out-patients. Br Dent J 1984;156: 261.

3. Carter L M, Godlington F L, Meechan J G. Screening for hypertension in dentistry. J Dent Res 1997; 76: 1037 Abstract 152.

4. Perusse R, Goulet J-P, Turcotte J-Y. Contra-indications to the use of vasoconstrictors in dentistry. Part I. Oral Surg 1992; 74: 679-686.

5. Seymour R A, Lowry R, Whitworth J M, Martin M V. Infective endocarditis, dentistry and antibiotic prophylaxis; time for a rethink? Br Dent J 2000; 189: 610-616.

6. Martin M V , Gosney M A, Longman L P, Figures K H. Murmurs, Infective Endocarditis and Dentistry. Dent Update 2001; 28 No.2: 76-82

7. Dessler FA, Roberts W C. Mode of death and type of cardiac disease in opiate addicts: analysis of 168 necroscopy cases. Am J Cardiol 1989; 64: 909-920.

8. Ryder W. The electrocardiogram in dental anaesthesia. Anaesthesia 1970; 25: 46-62.

9. Thomason J M, Seymour R A, Ellis J S, Kelly P J, Parry G, Dark J. latrogenic gingival overgrowth in cardiac transplantation. J Periodontol 1995; 66: 742-746

10. Meechan J G, Thomason J M, Rattray D T, Parry G. The use of dental local anaesthesia in cardiac transplant recipients. J Dent Res 1997; 76: 154 Abstract.

11. Gilbert E M, Eiswirht C C, Mealey P C, Larrabee B S, Herrick C $M$, Bristow M R. B-adrenergic supersensitivity of the transplanted heart is pre-synaptic in origin. Circulation 1989; 79: 344-349.

12. Sugimura $M$, Hirota $Y$, Shibutani $T$, Xiwa $H$, Hori $T$, Kim $Y$, Matsuura $H$. An echocardiographic study of interactions between Pindolol and epinephrine contained in a local anesthetic solution. Anesthesia Progress 1995; 42: 29-35.

13. Meechan J G. Plasma potassium changes in hypertensive patients undergoing oral surgery with local anaesthetics containing epinephrine. Anesthesia Progress 1997; 44: 106-109. 